\title{
The Concept of Spatial Channel Theory Applied to Reactor Shielding Analysis
}

\author{
M. L. Williams \\ W. W. Engle, Jr.
}

\section{OAK RIDGE NATIONAL LABORATORY}




\section{DISCLAIMER}

This report was prepared as an account of work sponsored by an agency of the United States Government. Neither the United States Government nor any agency Thereof, nor any of their employees, makes any warranty, express or implied, or assumes any legal liability or responsibility for the accuracy, completeness, or usefulness of any information, apparatus, product, or process disclosed, or represents that its use would not infringe privately owned rights. Reference herein to any specific commercial product, process, or service by trade name, trademark, manufacturer, or otherwise does not necessarily constitute or imply its endorsement, recommendation, or favoring by the United States Government or any agency thereof. The views and opinions of authors expressed herein do not necessarily state or reflect those of the United States Government or any agency thereof. 


\section{DISCLAIMER}

Portions of this document may be illegible in electronic image products. Images are produced from the best available original document. 
Printed in the United States of America. Available from National Technical Information Service

U.S. Department of Commerce

5285 Port Royal Road, Springfield, Virginia 22161

Price: Printed Copy $\$ 4.00$; Microfiche $\$ 3.00$

I his report was prepared as an account of work sponsored by an agency of the United States Government. Neither the United States Government nor any agency thereof, nor any of their employees, contractors, subcontractors, or their employees, makes any warranty, express or implied, nor assumes any legal liability or responsibility for any third party's use or the results of such use of any information, apparatus, product or process disclosed in this report, nor represents that its use by such third party would not infringe privately owned rights. 
Contract No. W-7405-eng-26

Neutron Physics Division

THE CONCEPT OF SPATIAL CHANNEL THEORY APPLIED TO REACTOR SHIELDING ANALYSIS*

M. L. Williams and W. W. Engle, Jr.

Manuscript Prepared By

Susan Y. Marler

Juily, 1976

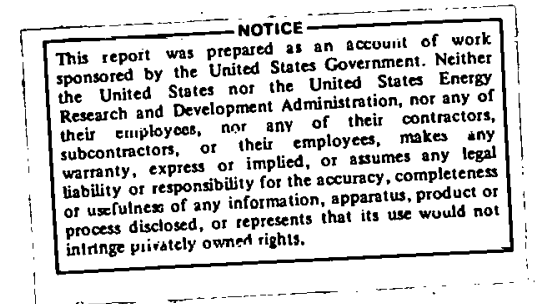




\section{THIS PAGE}

WAS INTENTIONALLY

LEFT BLANK 
TABLE OF CONTENTS

Page

Abstract ...................... . . . . vii

Introduction . . . . . . . . . . . . . . . . . . 1

Theory . . . . . . . . . . . . . . . . . . 4

Example . . . . . . . . . . . . . . . . . . 17

Practical Application . . . . . . . . . . . . . . . 23

Conclusion . . . . . . . . . . . . . . . . . . . . 31

Acknowledgements. . . . . . . . . . . . . . . 32

References . . . . . . . . . . . . . . . . 33 


\section{THIS PAGE \\ WAS INTENTIONALLY \\ LEFT BLANK}


Fig. 1. Cutaway View of Fast Test Reactor and Surrounding Regions. . . . . . . . . . . . . . . . . . . . . .

Fig. 2. Example Problem One Used in Channel Theory Calculations . . . . . . . . . . . . . . . . . .

Fig. 3. Perspective Plot of Contributon Flux in Example Two.

F1g. 4. Geumeliy for step 1 of R-Z Calculatione of FFTF. .

Fig. 5 \& 6 . Geometries for Steps 2 and 3 of R-Z Calculations of FFTF. . . . . . . . . . . . . . . .

Fig. 7. Contributon Flux in RCS Region of FTR. . . . . . .

Table 1. Contributon Leakage Integrated Over $X$ in Example Problem One. . . . . . . . . . . . . . . . . . . . .

Table 2. Contributon Leakage Integrated Over $r$, in FFTF RCS Geometry. 


\section{THIS PAGE}

\section{WAS INTENTIONALLY \\ LEFT BLANK}




\section{ABSTRACT}

The concept of Channel Theory is used to locate spatial regions which are important in contributing to a shielding response. The method is analogous to the channel theory method that was developed for ascertaining important energy channels in cross-section analysis. The mathematical basis for the theory is shown to be the generalized reciprocity relation, and sample problems are given to exhibit and verify the properties which are predicted by the mathematical equations. Finally, a practical example is cited from the FFTF shielding analysis performed at Oak Ridge, in which a perspective plot of channel theory results was found useful in locating streaming paths around the reactor cavity shield. 


\section{INTRODUCTION}

Until relatively recent years, reactor shielding computation was limited to uncomplicated models, many times one-dimensional in nature, or at least containing simplifying assumptions in a geometrical mock-up of the physical system. With the advent of larger and faster computers, as well as improvements in discrete ordinates, Monte Carlo, and coupled

techniques, ${ }^{1}$ it is now common to calculate responses in systems of extremely rnmplex geometries. As an example, Fig. 1 shows a portion of the Fast Flux Test Facility Reactor, which was modeled in discrete ordinates shielding calculations at ORNL.

In today's detailed analyses requiring multi-dimensional geometries with multiple streaming paths, it is many times difficult to identify the important regions that significantly contribute to the response of interest; and to thus locate the most effective shield location. Determination by trial and error is usually not acceptable due to the high cost of each calculation.

For this reason, a technique known as channel theory has been developed, which effectively calculates important "channels" that contribute to the response; e.g., it locates pertinent streaming paths. The related concept of an "energy channel theory" was suggested by Goldstein, et a ${ }^{2}$ to investigate important cross-section channels through which particles flow to affect a particular response. The energy paths can then be employed to ascertain the sensitivity of the response to cross-section properties. The present theory is analogous, except that it is used to determine the sensitivity of the response to geometrical properties of the reactor. This "spatial channel theory" evolved from earlier work 


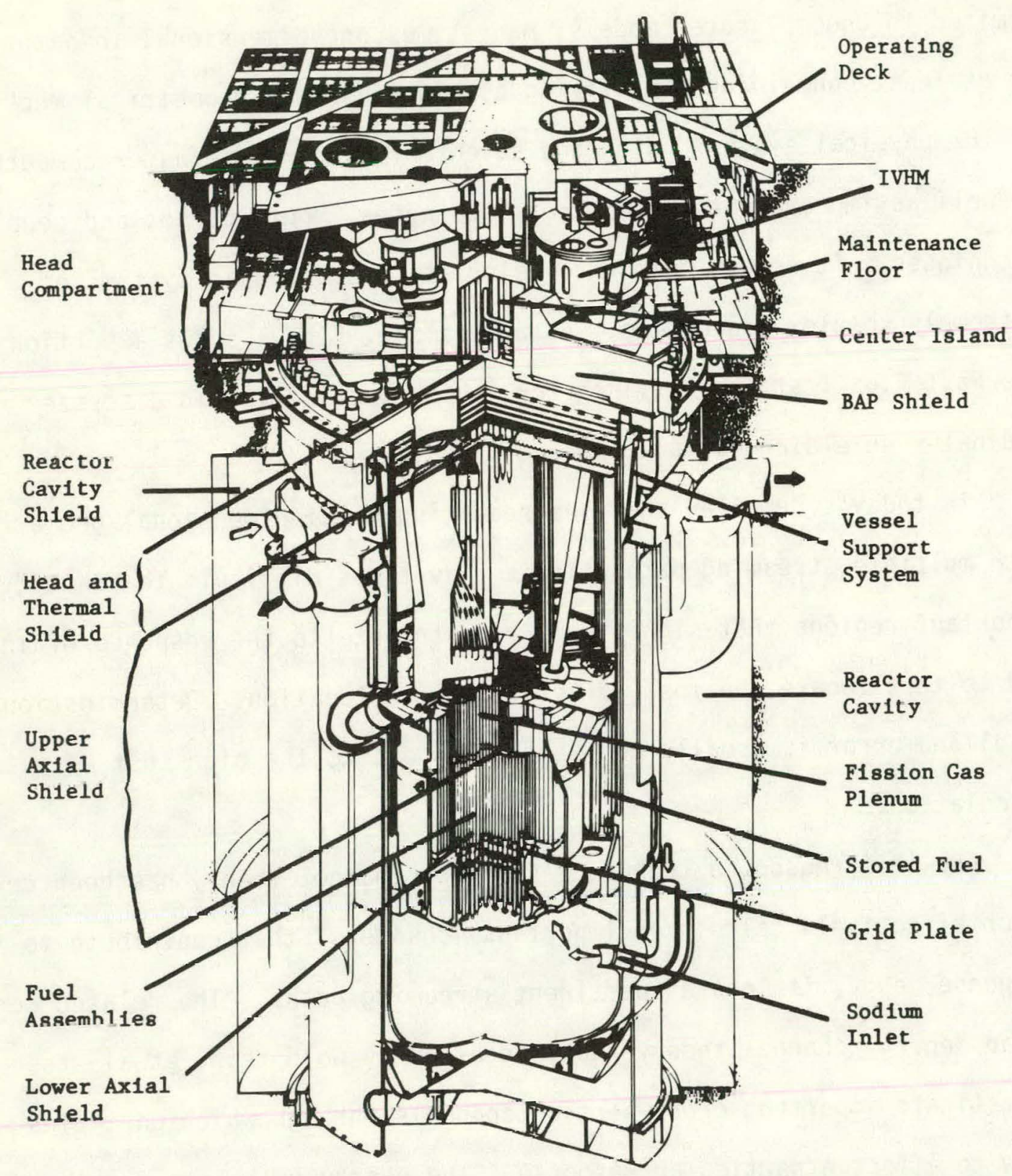

Fig. 1. Cutaway V1ew of Fust Test Reactur and Surrounding Regions. 
done at Oak Ridge during the SNAP reactor program, where simple forwardadjoint folding was applied to the optimization of a shadow shield. ${ }^{3}$ The technique was made possible by the development of the $\mathrm{FACT}^{4}$ code, which provided for the coupling of dissimilar (in mesh size and quadrature) forward and adjoint calculations at some particular surface. The present work extends the application of channel theory to two space dimensions and also investigates some of its theoretical implications.

In the next section, the spatial theory is developed and shown to predict useful properties for application to radiation transport problems. Following that section are two illustrative examples which are representative of the type to which channel theory can be applied. The final section contains a more complex problem where spatial channel theory was actually used in an FFTF shield analysis. 


\section{THEORY}

In a mathematical sense, a reactor is merely some closed region in a multi-dimensional phase space. Reactor physics and shielding calculations are deeply involved in determining a quantity known as the "particle distribution" throughout that particular phase space volume. However, a very important, though subtle, point which must be realized is that the particle distribution itself can never be observed; only the effects of the distribution are observable. The observable may be a temperature reading, an electrical impulse registered on a meter, or dny number of other quantities, either pointwise or integral; but in every case, it is only a manifestation of some portion ur the particle distribution which is perceived.

One can imagine a second, independent distribution which relates the effect that the particle distribution at each point in phase space has upon the observable of interest. Alone, each of these distributions -the particle and the effect -- is imperceptible to the senses, and it is unly the combination of the two functions which has physical significance. The "effect distribution" is well known to be the adjoint function to the particle distribution. ${ }^{5}$

The above discussion sontains the fundamental concept for cladmel theory; only observable quantities are physical; and these observables are affected by unique subsets of a mathematical function called the particle flux. Each subset is related to its respective observable by a second mathematical function called the adjoint. flux. The inter-rclation between these two functions will now be examined. 
In any reactor region, the forward and adjoint fluxes must satisfy the particle transport equation throughout the phase space volume and must satisfy boundary conditions at the surface.

$$
\begin{aligned}
& \operatorname{L\phi }(\vec{\rho})=Q(\vec{p}) \\
& L_{\phi}^{+}(\vec{p})=Q^{+}(\vec{p}) \quad, \operatorname{\rho \varepsilon v}(\vec{p}) \\
& \phi_{S}=\text { B.C. } \\
& \phi_{S}^{+}=\text {B.C. } .^{+}, \text {AT SURFAC }
\end{aligned}
$$

where $v$ is appropriate phase space volume,

$L$ is a transport operator, and

$\mathrm{L}^{+}$is its adjoint operator.

$\vec{o}=$ vector in phase space.

$Q=$ fixed, forward source.

$Q^{+}=$. fixed, adjoint source.

For the particular case of $L$ being the time-independent Boltzmann operator for a non-multiplying medium (as is usually encountered in shielding calculations) the transport equation becomes

$$
\begin{aligned}
& \hat{\Omega} \cdot \hat{\nabla}_{\phi}(\vec{\rho})+\Sigma_{t^{\phi}}(\vec{p})= \\
& \int_{E^{\prime} \cdot \int_{\Omega^{\prime}}} \sum_{S}\left(\vec{r}, E^{\prime}, \Omega^{\prime} \rightarrow E, \Omega\right) \phi\left(\vec{r}, E^{\prime}, \Omega^{\prime}\right) d \Omega^{\prime} d E^{\prime}+Q(\vec{p}) \\
& \vec{r}=\text { spatial variables in phase space. }
\end{aligned}
$$


and its adjoint equation is

$$
\begin{aligned}
& -\hat{\Omega} \cdot \hat{\nabla}_{\phi}^{+}(\vec{\rho})+\Sigma_{t^{\phi}}{ }^{+}(\vec{\rho})= \\
& \quad \int_{E^{\prime}} \int_{\Omega^{\prime}} \Sigma_{S}\left(\vec{r}, E, \Omega \rightarrow E^{\prime}, \Omega^{\prime}\right) \phi^{+}\left(\vec{r}, E^{\prime}, \Omega^{\prime}\right) \mathrm{d} \Omega^{\prime} \mathrm{dE} E^{\prime}+Q^{+}(\vec{\rho})
\end{aligned}
$$

One can obtain a "pointwise reciprocity theorem" by multiplying the forward equation by $\phi^{+}$and the adjoint equation by $\phi$, suhtracting the two equations, and then integrating over only the energy and angular phase space variables.

$$
\begin{gathered}
\int_{E} \int_{\Omega} \phi^{+}(\vec{\rho}), \dot{\Omega} \cdot \nabla \phi(\vec{\rho}) \mathrm{d} \Omega \mathrm{dE}+\int_{\mathrm{E}} \int_{\delta_{0}} \phi(\vec{\rho}), \hat{\Omega} \cdot \nabla \phi+(\vec{\rho}) \mathrm{d} \Omega \mathrm{dE}= \\
\int_{E} \int_{\Omega}\left[\phi^{+}(\vec{\rho}), Q(\vec{\rho})-\phi(\vec{\rho}), Q^{+}(\vec{\rho})\right] \mathrm{d} \Omega \mathrm{dE}
\end{gathered}
$$

Note that the $\Sigma_{t}$ and the scattering terms vanish due to the properties of the adjoint operator. Eq. (7) can be rewritten as

$$
\widehat{\operatorname{div}} \int_{E} \int_{\Omega} \hat{\Omega}_{\Omega} \phi^{+} \mathrm{d} \Omega \mathrm{dE}=\int_{E} \int_{\Omega}\left(\phi^{+}, Q-\cdot \phi, Q^{+}\right) \mathrm{d} \Omega \mathrm{dE}
$$

Eq. (8) is the governing theorem for channel theory, and its implications will be discussed shortly.

The more familiar form of the reciprocity equation, which will be referred to as the "global reciprocity equation," can be easily derived from Eq. (8) by integrating over the spatial variables and applying Gauss' theorem to the divergence term: ${ }^{6}$ 


$$
\left\langle\phi_{\phi}^{+}(\vec{\rho}), Q(\vec{\rho})-\phi(\vec{\rho}), Q^{+}\left(\begin{array}{l}
0 \\
0
\end{array}\right)=\oint_{A} \mathrm{dA} \int_{E} \int_{\Omega} \hat{n} \cdot \hat{\Omega} \phi \phi{ }^{+}(\vec{\rho}) \mathrm{d} \Omega \mathrm{dE}\right.
$$

where

$\hat{n}$ normal to $d A$.

\langle\rangle indicates integration over all phase space variables.

It is obvious that if the incoming flux and the outgoing adjoint at the surface are equal to zero, the surface term vanishes; therefore, for the case of vacuum boundary conditions, the reciprocity theorem reduces to

$$
\left\langle{ }_{\phi}^{+}(\vec{p}), Q(\vec{p})\right\rangle=\left\langle\phi(\vec{p}), Q^{+}(\vec{p})\right\rangle, \quad,
$$

with the auxiliary condition

$$
\left\langle\phi(\vec{p}), Q^{+}(\vec{p})\right\rangle=R[\phi],
$$

where $R[\phi]$ is some response to be observed.

These equations are used frequently in shielding calculations whenever a response must be calculated for a variety of source conditions, because they give the response functional in terms of the adjoint flux, and thus eliminate the need for new forward calculations. Since most shielding and reactor physics problems can be represented with vacuum boundary conditions (reflected can be reduced to a vacuum), Eq. (10) is the form of the reciprocity theorem most familiar to nuclear engineers. However, channel theory calculations will be based on the use of the general theorem for the following reason: The global reciprocity theorem relates integral quantities; it contains a volume integral over 
the source regions and a surface integral over the closed boundary surrounding the phase space volume. To obtain any information about an interior point of the geometrical phase space volume, such as location of important streaming paths, the interior point must be related to one of these integral quantities. This can be done by defining an equivalent problem [i.e., one that produces the same $\phi(\overrightarrow{0})]$ in which the interior point of interest lies on the surface of a sub-domain of the original phase space volume. The fluxes in this sub-domain will be identical to those in the original problem if the angular fluxes along the common boundary of the two regions are equal, a fact which fixes the boundary conditions for the new region. An illustration at this point will clarify the procedure.

Consider the following $X Z$ geometry frequently encountered in shielding calculations

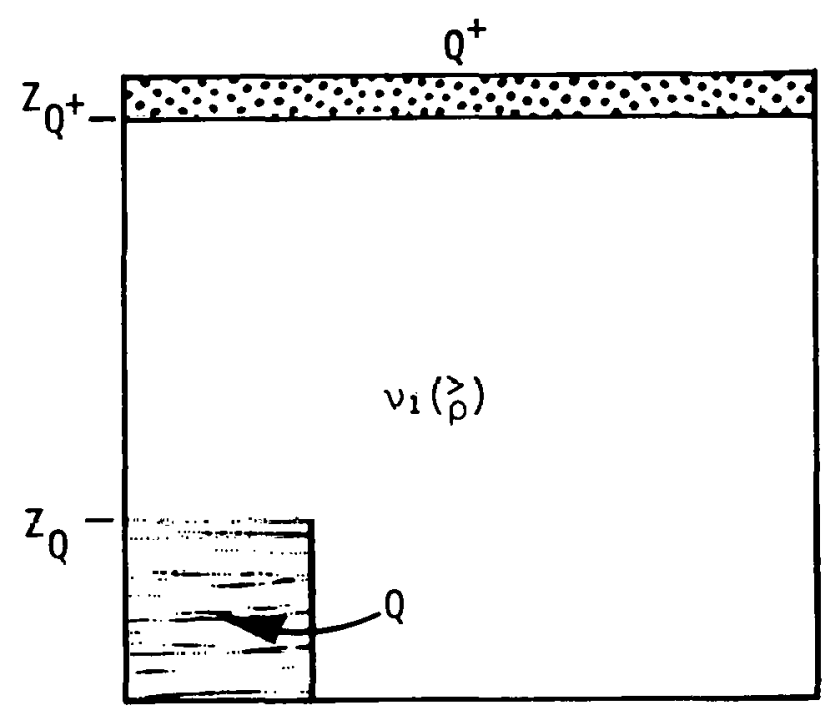

To be specific, assume that $Q$ is a volume distributed source corresponding to a reactor core, and $Q^{+}$is a flat, volume distributed adjoint source with a spectrum equal to the flux-to-dose conversion factors. Therefore, the appropriate response for this example is 
$R[\phi]=\left\langle\phi(\vec{p}), Q^{+}(\vec{p})\right\rangle=$ integrated dose in dotted region.

The remaining, unshaded portion of $v(\vec{p})$ is in general a geometrically complex region, containing structure, pipes, control systems, shielding, etc.

Suppose that it is desired to identify the contribution to the observable per unit area along some arbitrary height $z_{0}$ within $v_{1}(\dot{\rho})$. To do this, first define the following separate but associated problem.
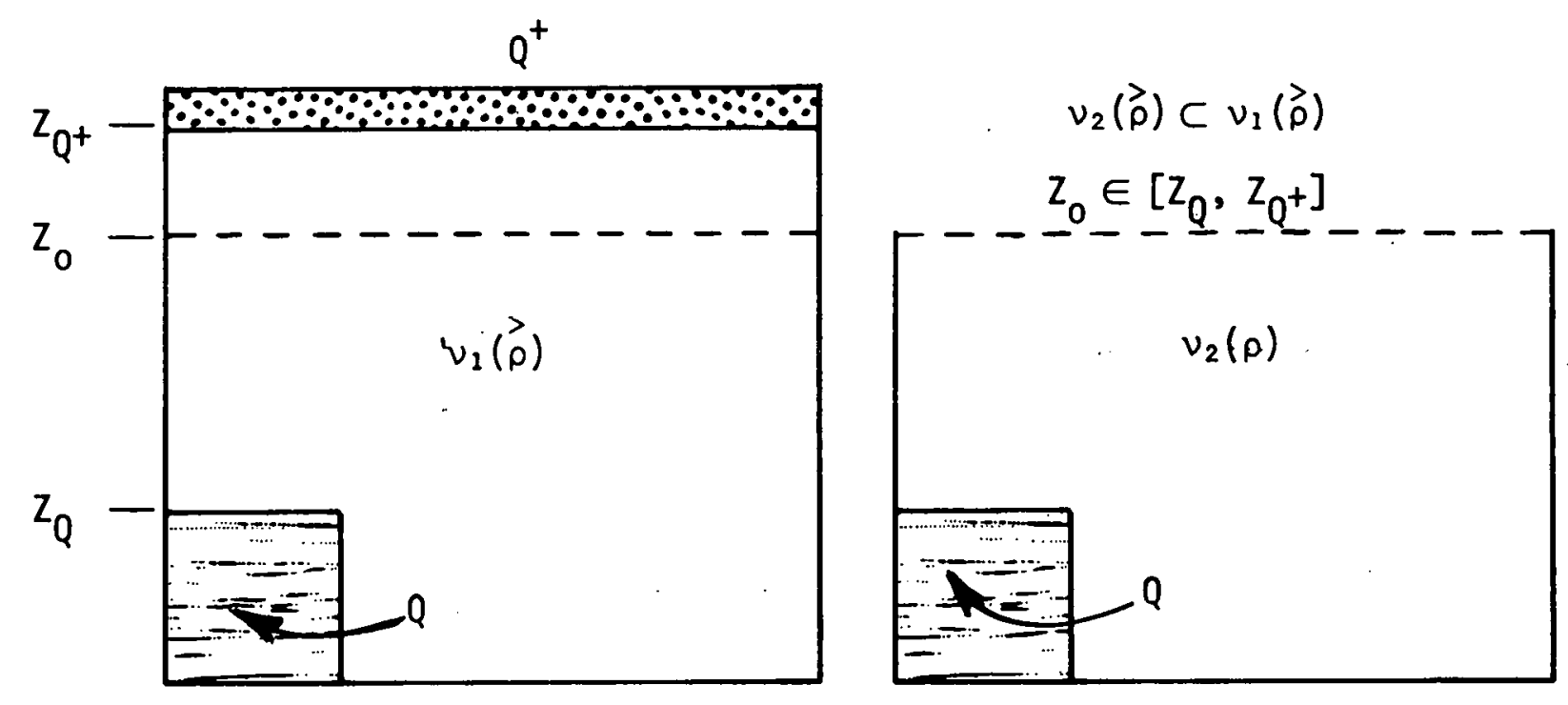

The forward and adjoint fluxes calculated in $v_{2}$ will be identical to those in the corresponding $v_{1}$ region if the boundary conditions of along the dotted top boundary are made equal to the angular forward and adjoint fluxes at $z_{0}$ in $v_{1}$.

Now consider the global reciprocity relation in both of these regions. For $v_{1}$, with var.uum boundary conditions, it is

$$
\left\langle\phi, Q^{+}\right\rangle=\left\langle\phi^{+}, Q\right\rangle=R[\phi]
$$


This merely illustrates the earlier statement that folding an adjoint flux with a forward source will give a desired response without requiring a forward flux calculation.

The second region contains no adjoint source, and it no longer has a vacuum boundary condition along the top surface. Therefore, the reciprocity relation becomes

$$
\begin{aligned}
\left\langle\phi^{+}, Q\right\rangle & =\left\langle{ }_{\phi} Q^{+}\right\rangle=\oint_{A}[\text { Surface Terms }] d A \\
& =\int_{\substack{\text { Top } \\
\text { Boundary }}}[\text { Surface Terms }] d A .
\end{aligned}
$$

Substituting the value in Eq. (9) for the surface integration and setting the top boundary fluxes. in $v_{2}$ equal to the fluxes at $z_{0}$, in $v_{1}$,

$$
\int_{Z=Z}\left\{\int_{E} \int_{\Omega}(\hat{n} \cdot \hat{\Omega})_{\phi}(\Omega, E, Z, X)_{\phi}^{+}(\Omega, E, Z, X) d \Omega d E\right\} d A=\left\langle\phi, Q^{+}\right\rangle
$$

The term in brackets in Eq. (14) will now be written in terms of a new function defined by

$$
\hat{D}(X, Z)=\int_{E} \int_{\Omega} \hat{s} \phi\left({ }^{\prime}\right) \phi_{\phi}^{+}(\vec{\rho}) \mathrm{d} \Omega \mathrm{d} E
$$

This vector function is the basis for channel theory and will be shown to be the "current" of a special particle which represents the contribution of a point $(X, Z)$ to the specified observable, $R[\phi]$. The special particle will have a "scalar flux" given by 


$$
C(X, Z)=\int_{E} \int_{\Omega} \phi(\vec{\rho}) \phi^{+}(\vec{\rho}) \mathrm{d} \Omega \mathrm{dE}
$$

Notice that. Eq. (14) produces the following interesting result:

$$
\int_{A} \hat{n} \cdot \hat{D}(X, Z) d A=R[\phi]
$$

for $Z=Z_{0}$

Since $z_{0}$ was chosen arbitrarily, one concludes that integration of $\hat{n} \cdot \hat{D}(x, Z)$, which is merely the leakage of $C(x, Z)$, over any surface corresponding to a horizontal slice through the reactor, lying between the forward and adjoint sources, will give the value of the response functional, a constant. For this particular example, the leakage in the $Z$ direction was observed, but other components could also have been calculated by using differently directed unit vectors for $\hat{n}$; and in general, Eq. (14) can be written as:

$$
\oint_{S} \hat{n} \cdot \hat{D}(x, Z) d S=R[\phi]=\oint_{S}(\hat{n} \cdot \hat{e})|\hat{D}(x, Z)| d S
$$

where

$\hat{e}$ is unit vector in direction of $\hat{D}(x, z)$

$S$ is any surface surrounding the forward or adjoint sources

$\hat{n}$ is unit vector normal to dS

Intuitively, it is obvious that along a constant $z$ level, the leakage of $C(X, Z)$ in the $Z$ direction will be some function of $X$, having a maximum value at the location of greatest contributon to $R[\phi]$ due to particles 
traveling in the $Z$ direction. This coordinate value will be referred to as $R_{\max }(Z)$. A plot of $R_{\max }(Z)$ will locate the path followed by particles whose $\mathrm{Z}$ component of transport provides the largest contribution to the observable $R[\phi]$; i.e., the major axial streaming path. Similar arguments apply to values other than the maximum value.

Usually the direction in which a contributing particle is traveling does not interest a designer; he only cares about its final contribution. To observe this quantity, one should not look at one particular vector component of the current $\dot{D}(X, Z)$, but instead must consider its total track length magnitude, which is merely $C(x, z)$. This function, then, will give the contribution due to all the particles present at $(X, Z)$, regardless of their direction of propagation, and a mapping of $C(X, Z)$ should reveal the relative contribution at each coordinate position. Now that the function $\hat{D}(X, Z)$ has been identified, several corollary observations should be made.

First, if the "associated problem" defined in deriving $\hat{D}(X, Z)$ was chosen to be the region above $z_{0}$, instead of the region below it, the following procedure would have been followed.
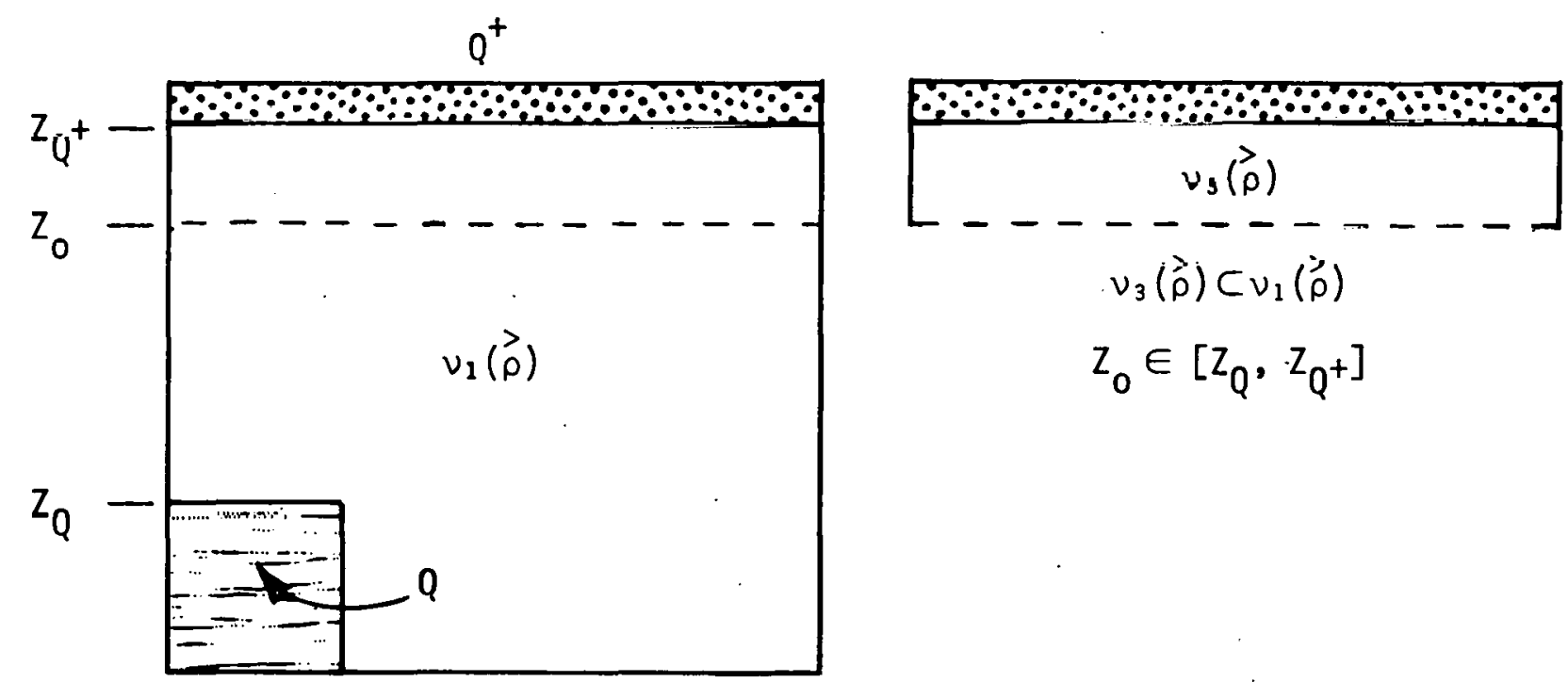
Make the boundary condition at the bottom boundary of $v_{3}$ equal to the angular flux at $z_{0}$. Then the global reciprocity theorem is

$$
\begin{aligned}
& -\left\langle{ }_{\phi, Q^{+}}\right\rangle=\oint[\text { Surface Terms }] \mathrm{dA} \\
& \mathrm{d} \\
& \left\langle\phi, Q^{+}\right\rangle=R[\phi]=-\int_{X} \hat{n} \cdot \hat{D}(X, Z) d X
\end{aligned}
$$

Therefore, if the alternate "associated problem" had been assumed, its only effect on the final conclusion would be a change of sign.

Physically, this has the same effect as interchanging the forward and adjoint sources; a process which reverses the flow of $\hat{D}(X, Z)$.

The first property discussed is reassuring since it suggests that the conclusions derived are independent of coordinate orientation, a fact which is physically obvious.

Second, consider a surface which lies within a volume distributed source:
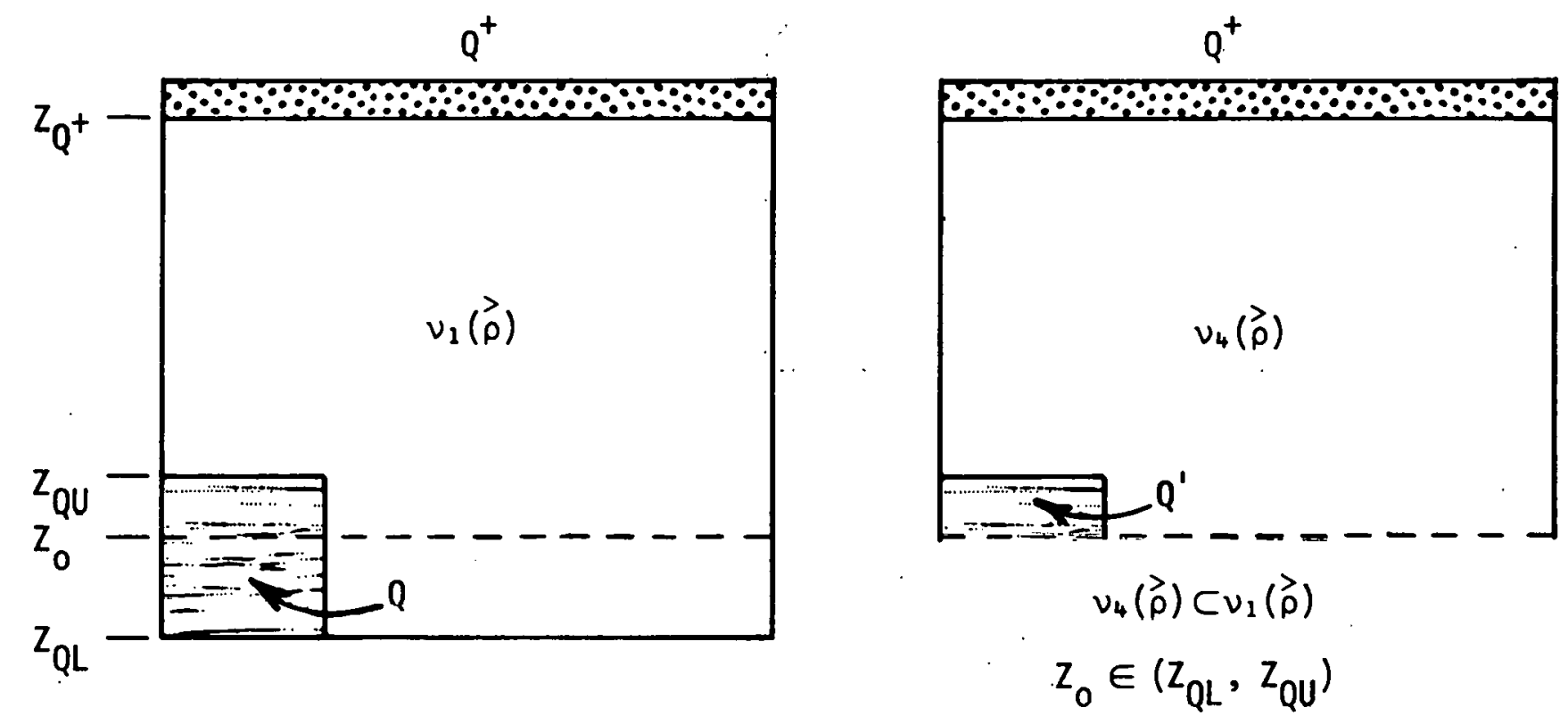
The reciprocity equation is

$$
\int_{x} \hat{n} \cdot \hat{D}(x, Z) d x=-\left\langle\phi^{+}, Q^{-}\right\rangle-\left\langle\phi, Q^{+}\right\rangle
$$

where $Q^{\prime}$ is now only that portion of the forward source which lies above $\mathrm{z}_{0}$.

For this case, the integrated value of $\hat{D}(X, Z)$ gets larger as $Z_{0}$ approaches $L_{Q u}$, becoming equal to the response when $Z=Z_{Q u}$. At that point, it remains constant, as previously discussed. Similar results occur if $z_{0}$ is chosen to $1 \mathrm{ie}$ withth the adjoint sourcc.

Third, if a surface does not lie in the closed interval of the forward and adjoint sources, the following example is applicable.
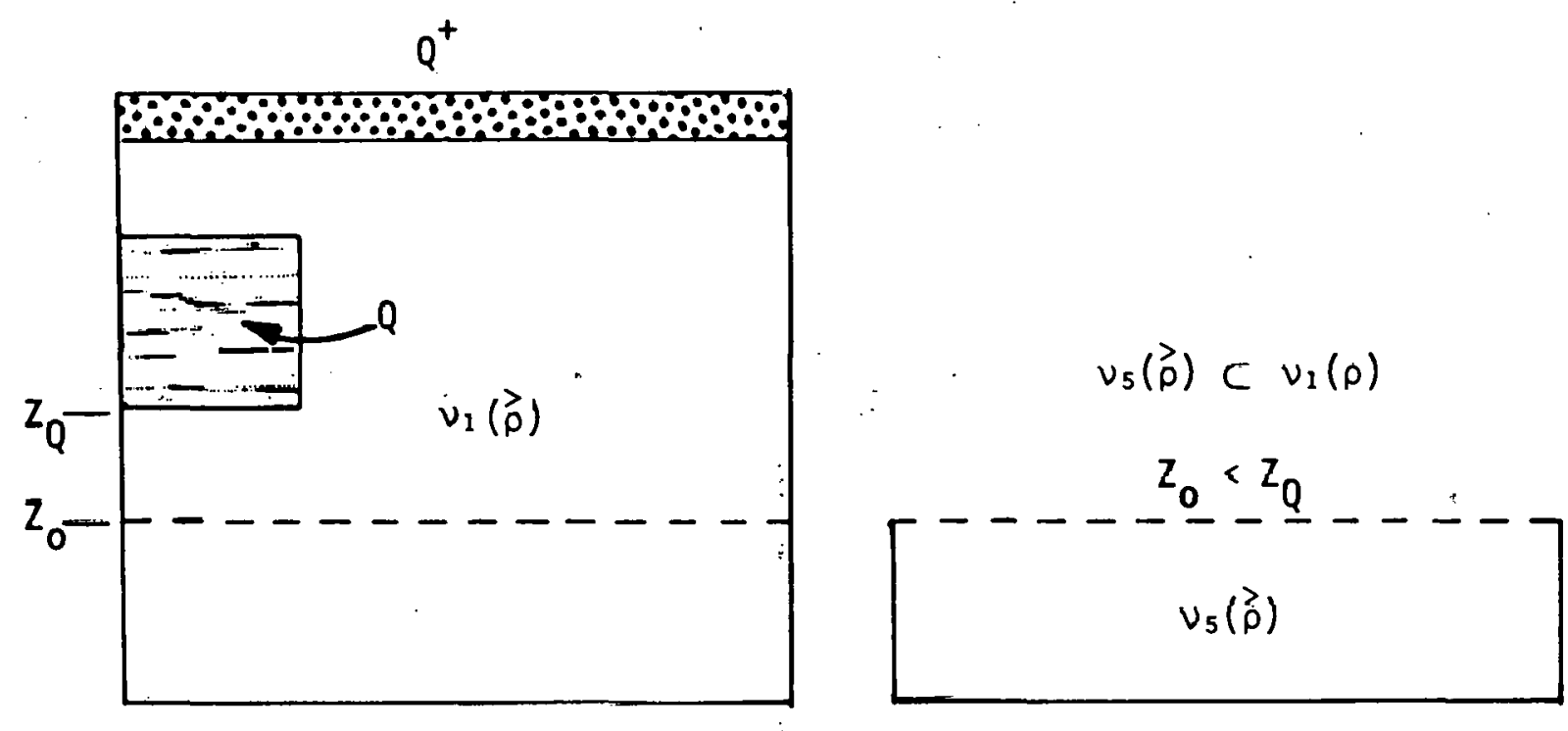

Since the associated problem now contains neither forward nor adjoint sources, the appropriate reciprocity relation is 


$$
\begin{aligned}
\int \hat{n} \cdot \hat{D}(x, z) d x & =0, \\
z_{0}< & z_{Q} \\
& \text { for } z=z_{0}
\end{aligned}
$$

Thus, the surface integral vanishes if a source is not contained within the enclosed volume. A physical explanation for this phenomenon is qiven shortly.

Finally, the function $C(X, Z)$ has an interesting physical interpretation. It can be viewed as the spatial distribution for the flux of a special kind of particle called a "contributon" that represents only those particles which actually contribute to the observable. This flux will be referred to as the "contributon flux" or synonymously, as the "response flux", for obvious reasons. The $\hat{D}(X, Z)$ function (Eq. 15) will be called the "contributon current": As indicated in discussions above, it should be clear that these contributons can never be lost. They can change position, direction, and energy, but they will aiways eventually fulfill their mission of contributing to the response. Since the particles are never lost, any contributons emitted from the forward source must necessarily pass through a closed surface cutting the reactor between the forward and adjoint sources (actually, it is more accurate to say they must pass through the surface an odd number of times, to account for back scattering). Therefore, integration of the contributons leakage over one of these surfaces will yield the total contribution, which is the observable $R[\phi]$. On the other hand, a contributon emitted from the forward source must pass through a surface that does not lie between the two sources an even number of times (once going out and the 
next time coming back) in order to arrive at the adjoint source. This cancelling effect produces a zero response when the vector $\hat{D}(X, Z)$ is integrated over a surface not lying between the forward and adjoint sources.

The above physical interpretation which led to the concept of a response-flux can be easily explained mathematically. Without loss of generality, the flux-variable solution space can be expressed as the union of two sub-spaces: the flux arising from particles that contribute to the response and the flux from the other particles. The transformation on $\phi(\vec{\rho})$ that is implied by Eq. (16) allows one to enter the former sub. space; or, equivalently, it can be said that the transformation negates the latter sub-space. It kills (in a Monte Carlo sense) thuse particles not of interest. The flux is projected by the integral-adjoint operator onto a specific subset; and, with the transformation completed, only the response-flux can. be observed. Of course, this set comprised of contributons cannot be expected to have the same properties as the general flux set. In fact, it does not even obey the transport equation given by Eq. (5), Instead Eq. (8) can be thought of as the transport equation for the contributon flux:

$$
\nabla \cdot \hat{D}(\vec{r})=S(\vec{r})
$$

where $\hat{D}(\vec{r})=$ contributon current given by Eq. (15), and

$$
\begin{aligned}
S(\vec{r}) & =\int_{E} \int_{\Omega_{\iota}}\left[\phi^{+}(\vec{p}), Q(\vec{p})-\phi(\vec{\rho}) \cdot Q^{+}(\vec{n})\right] d \Omega \mathrm{dE} \\
& =\text { source of } \underline{\text { contributons }}
\end{aligned}
$$


Written in this form, the point reciprocity equation is merely the continuity equation for contributons. It differs from the Boltzmann transport equation by the absence of loss terms due to material interaction. This should be expected since contributons are never absorbed, and scattering events cannot be seen due to the integration over angle and energy.

\section{EXAMPLE}

The geometry shown in Fig. 2 was used to exemplify the channel theory concept. The model consisted of a square box of twenty-one $1 / 3$ $\mathrm{cm}$ intervals by seven $1 \mathrm{~cm}$ intervals, and contained in the center $\mathrm{Z}$ interval a forward source emitting 10 particles/sec $\mathrm{cm}^{3}$ and an adjoint source equal to 1 along the top interval. This adjoint source will give a response functional equal to the cell-centered forward flux in interval twenty-one. Reflected boundaries were used on the box's sides to avoid flux variation in the $X$ direction, and vacuum boundary conditions were used along the top and bottom. The box had a uniform composition, with a macroscopic $\sigma_{T}=1.0$ and $\sigma_{S}=.9$; the anisotropic component of the scattering cross section was 1.5 .

Forward and adjoint fluxes and flux moments were obtained for a one-group problem with the two-dimensional discrete ordinates code, DOT,? utilizing a $P_{1}$ Legendre expansion of the scattering cross section, a $S_{8}$ angular quadrature set, and a weighted difference flux approximation. Channel theory calculations were then performed with the computer code, FANG, ${ }^{8}$ which computes the function $\hat{D}(X, Z)$ by expanding DOT flux moments 


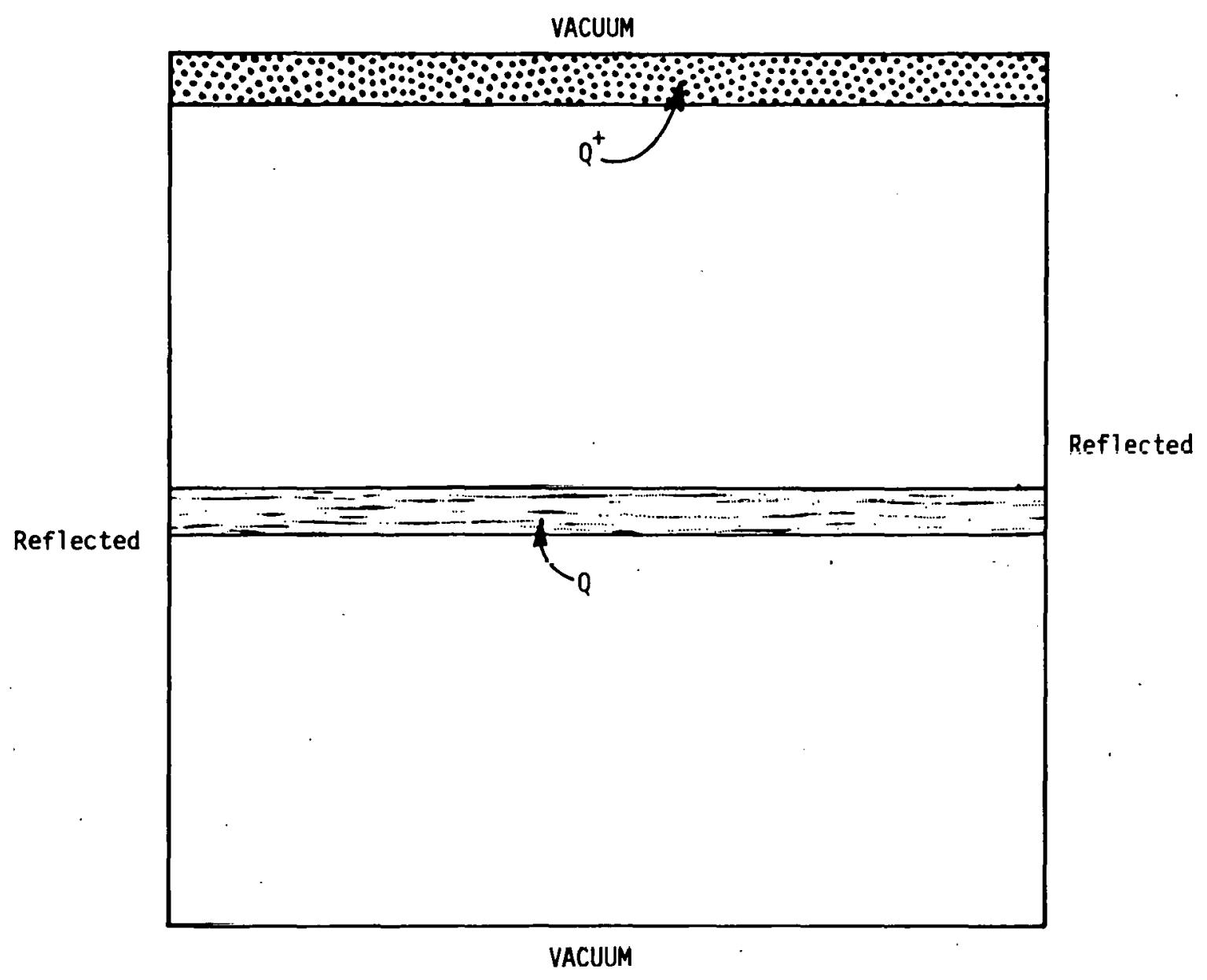

Fig. 2. Example Problem One Used in Channel Theory Calculation 
into angular fluxes and adjoints and then folding these with the appropriate angle. FANG uses a moments expansion for angular flux in the folding calculation, rather than directly folding the DOT angular flux, because in practical problems, the storage and retrieval of the angle dependent flux presents a significant data handling problem; and therefore, the flux moments are usually all that are retained in transport computations. . For this example, a $P_{5}$ expansion was used, although it was shown later that a $P_{3}$ spherical harmonic expansion gives adequate results even for the complex geometries described in the next section.

Table 1 shows the resulting $\hat{D}(X, Z)$ after it is integrated over $X$ for each axial interval to give the response $R[\phi]$. Because the region is infinite in the $X$ dimension, there is no variation of $\hat{D}(X, Z)$ in $X$, and the integrated values are proportional to $\hat{D}(X, Z)$ for a given $Z$. The table is divided into four groups corresponding to the ten intervals not located between the forward and adjoint sources, the interval containing the forward source, the nine intervals located between the sources, and the interval containing the adjoint source.

The values corresponding to the nine intervals lying between the forward and adjoint sources are approximately constant at 2.54. According to the predictions of the previous section, this should correlate to the response functional, which in this case is just the cell-centered flux integrated over the $X$ co-ordinate in Z-interval twenty-one. From the forward calculation, the flux at this position is found to be 1.08 particles $/ \mathrm{cm}^{2} \mathrm{sec}$; and, after a multiplication by the volume of $7 \mathrm{~cm} \times$ $.33 \mathrm{~cm}$, a response of 2.53 is obtained, which is within $.4 \%$ of the value found with channel theory. 
Table 1. Contributon Leakage Integrated Over $X$ in Example Problem One

\begin{tabular}{cc}
\hline Axial Interval & Response \\
\hline 21 & $1.08+00^{*}$ \\
20 & $2.53+00$ \\
19 & $2.54+00$ \\
18 & $2.54+00$ \\
17 & $2.54+00$ \\
16 & $2.54+00$ \\
15 & $2.54+00$ \\
14 & $2.51+00$ \\
13 & $2.54+00$ \\
12 & $2.53+00$ \\
11 & $1.18+00$ \\
10 & $3.07-03$ \\
9 & $6.84-04$ \\
8 & $2.62-04$ \\
7 & $2.13-04$ \\
6 & $1.43-04$ \\
5 & $8.74-05$ \\
4 & $5.17-05$ \\
3 & $3.01-05$ \\
2 & $1.74-05$ \\
1 & $1.12-05$ \\
& \\
\hline
\end{tabular}

*Rcad as $1.08 \times 10^{0}$. 
The two source intervals have integrated values lower than 2.54 . This is because DOT fluxes are evaluated at interval midpoints; and, therefore, the surface integration for $\hat{D}(X, Z)$ is actually over an area which lies within the Z-interval, and the "associated problem" must thus contain one-half interval of distributed source. As discussed in the "Theory" section, the presence of a distributed source will lower the response by an amount $\left\langle\phi^{+}, Q\right\rangle$; where, for this example, the limits of integration are over one-half the interval containing the source. For linear flux variations, this should lower the response by one-half; but, in this case it is not possible to exactly predict the reduction because the flux variation across an interval is not revealed in a DOT weighted difference calculation.

The remaining intervals are outside of the closed interval containing the forward and adjoint sources, and Table 1 shows that there is an abrupt decrease in the integrated values for these intervals. Of course, the magnitude does not drop to zero due to the numerical procedures employed, but a step-function drop of almost three orders of magnitude can be interpreted as verification that the $\hat{D}(X, Z)$ integral vanishes in this region.

A second example is illustrated by Fig. 3, which shows a perspective plot of the response-flux $C(X, Z)$, along with the geometry for which it was calculated. The bottom row of intervals contains a uniform, forward source of 10.0, and the top row contains an adjoint source identical to the forward source. The shaded areas in the geometry are highly absorbing regions, and the remainder is a predominately scattering medium. Forward and adjoint fluxes were found in the same manner as for Example One, and the folding was again accomplished with FANG. 

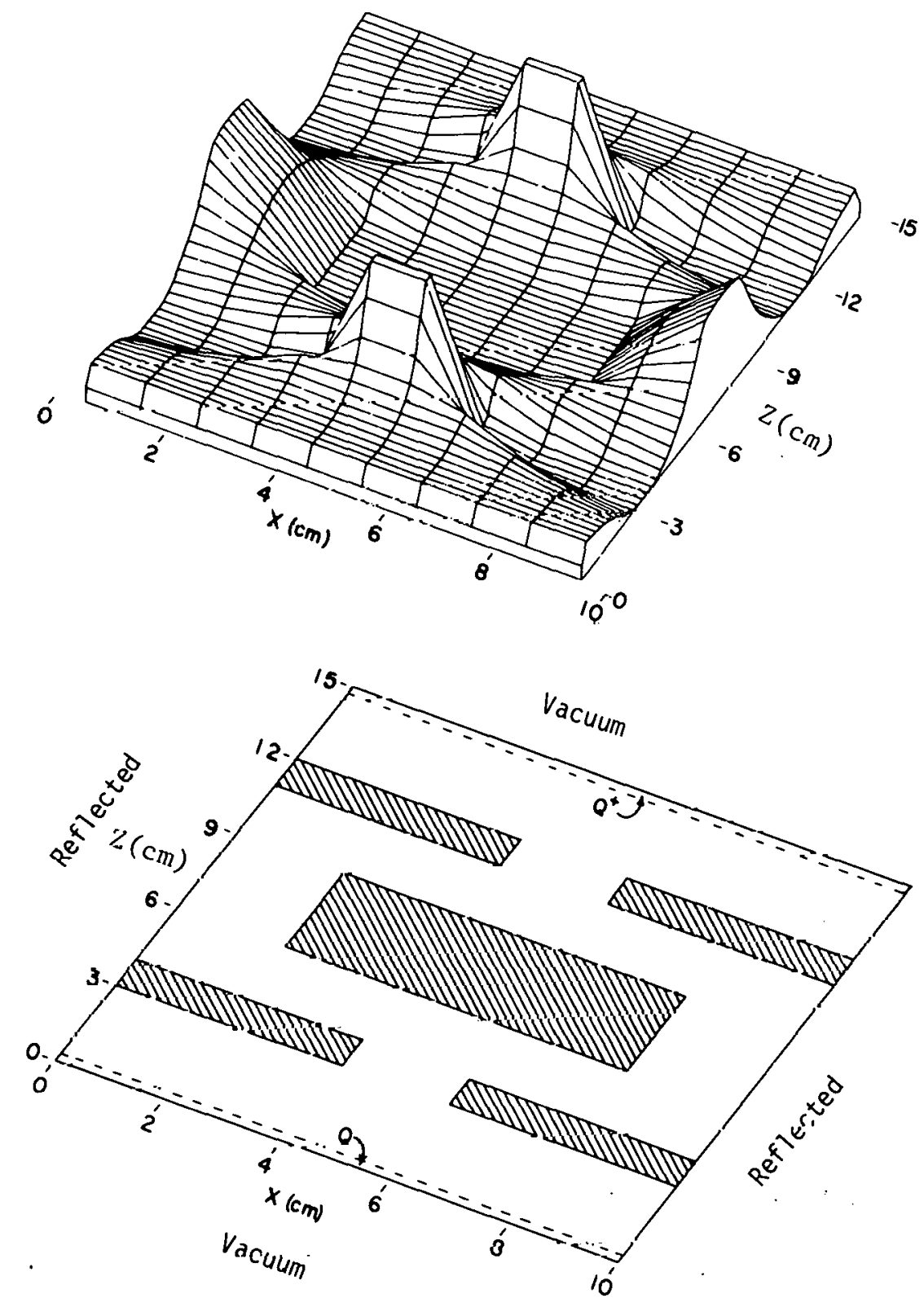

Fig. 3. Perspective Plot of Contributon Flux in Example Tro 
The plot of the response-flux clearly shows the origin of the name

"channel theory." The favored trajectories appear as ridges, whose altitudes are determined by their relative importance to the observed response. As expected, the contributons in this example flow around the absorbing regions, finding the channel of least resistance as they stream toward the adjoint source.

These simple examples prove some of the earlier statements asserted on theoretical grounds. The next section will show the practical merit for this type of analysis.

\section{PRACTICAL APPLICATION}

In the Fast Flux Test Facility shielding program at ORNL, calculations were performed to determine the dose rate during reactor operation in the region known as the head compartment, shown in Fig. 1. The calculations are quite formidable due to the great distance spanned, the presence of thick sections of stainless steel and sodium, and the existence of several narrow streaming paths. The only state-of-the-art. methods available which have hope of solving such a problem are. twodimensional discrete ordinates coupled with three-dimensional Monte Carlo, neither of which is able to perform independently the entire calculation in a reasonable manner. Adjoint discrete ordinates results were used to bias the Monte Carlo runs, which computed correction factors to account for the three-dimensional effects at strategic locations in the reactor. These correction factors were then applied to the forward, two-dimensional discrete ordinates calculations. 
To obtain enough spatial detail and remain within the limits of the ORNL IBM 360/91 computer core, it was necessary to break the discrete ordinates computation into three coupled $\mathrm{R}-\mathrm{Z}$ runs, a technique known as "bootstrapping." The geometries used for each of these calculations are shown in Figs. 4, 5, and 6 (coupling surfaces are given as dotted 1 ines). Fluxes for all three cases were calculated with DOT using a coupled 51neutron/25-gamma group cross-section library generated for FFTF studies by the AMPX 9 processing code.

The first step of the series used a $P_{1}$ cross-section expansion and a $S_{b}$ quadrature set. A rixed suurce was entered in the core and stored fuel regions, with a spatial distribution obtained from an earlier keigenvalue calculation $\left(P_{1}-S_{4}\right)$ of the FTR core. The remaining two steps of the computation were done with a $\mathrm{P}_{3}$ Legendre expansion of the scattering cross section and a biased 166 angle quadrature set.

The second step of the series centered around the area known as the Reactor Cavity Shield (RCS), in Figs. 1 and 5 . This reqion had earlier been identified as a significant streaming path for relatively high energy neutrons to enter the head compartment area. 10 For this reason, considerable effort went into studying the effects that changing shield composition and size, and enlarging streaming gaps had upon the biological dose in the head compartment.

During the course of the RCS design, a series of DOT calculations were made which used various types of concrete for the shield composition, and which enlarged by 1.5 inches the streaming gap between the RCS and the vessel support arm. The surprising result was that dose rates in the head compartment were not significantly affected by changes in RCS 


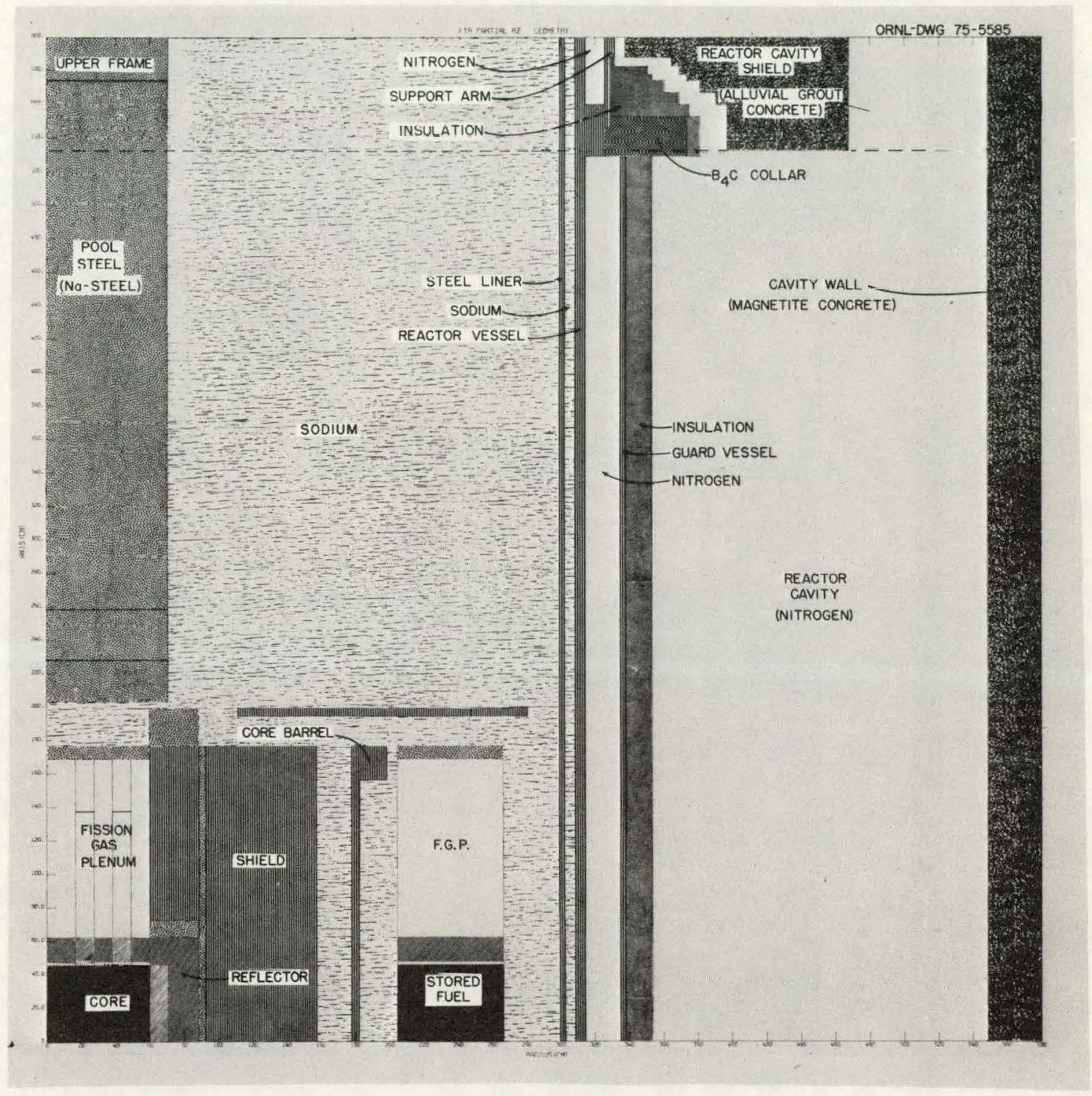

Fig. 4. Geometry for Step 1 of R-Z Calculations of FFTF. (Dashed line below reactor cavity shield indicates coupling surface.) 


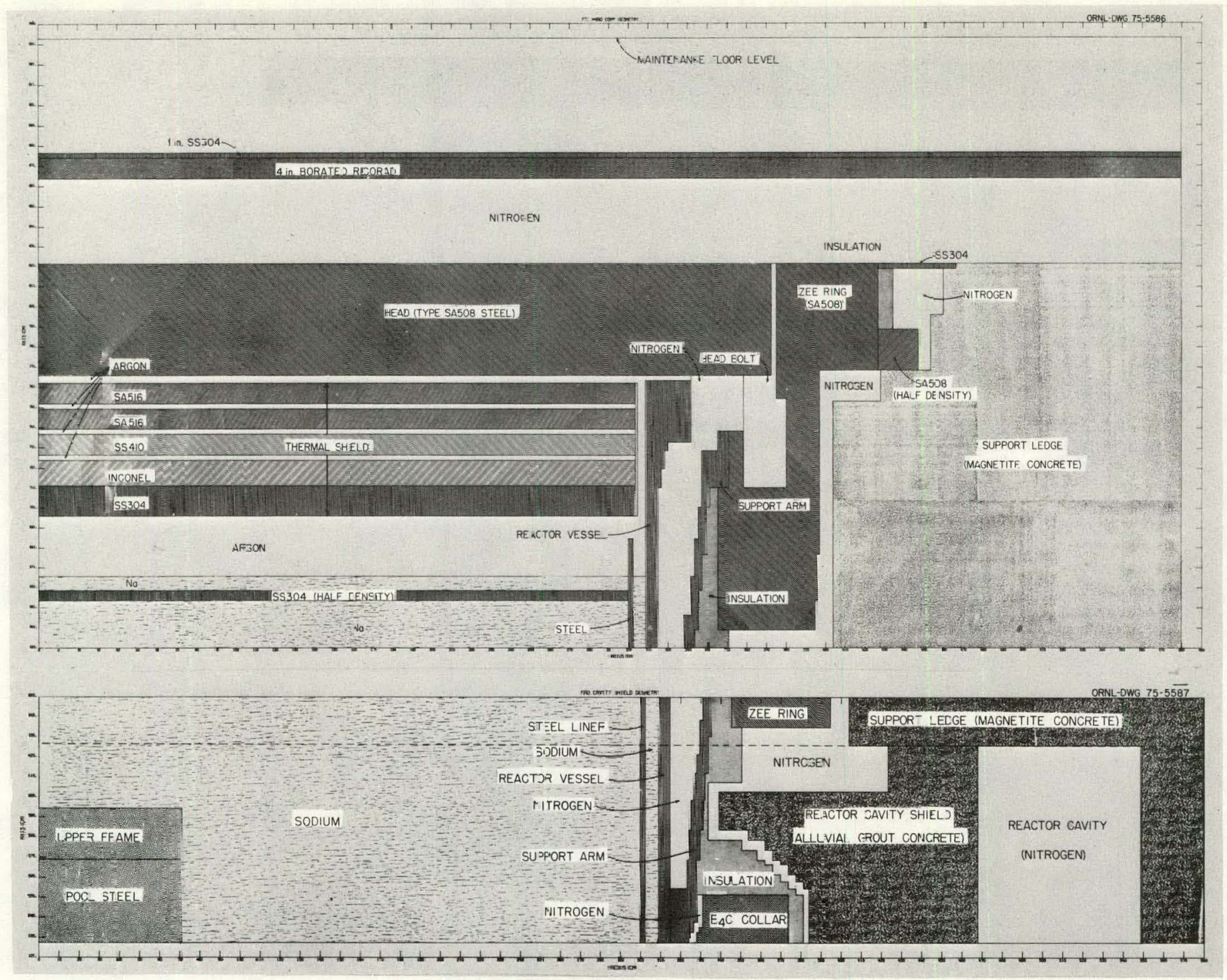

Figs. 5 and 6 . Geometries for Steps 2 and 3 of R-Z Calculations of FFTF. (Dashed lines indicates coupling surface) 
composition or in the streaming gap width. This was not fully understood, since it had been assumed that the major particle streaming was through the area around the sides of the $\mathrm{B}_{4} \mathrm{C}$ collar and up through the gap which now had been enlarged. If that were the case, however, an increase in the size of the streaming gap should cause a proportionate increase in the head compartment dose. Due to these unexpected results, it was next thought that the particles must flow to the inside of the flange connecting the vessel support arms to the vessel, and up through the sodium, where they entered the gap between the support arm and the vessel, and then streamed into the head compartment. This scenario was suggested because it was believed that significant particles could not penetrate the rather thick iron flange and directly enter the gap between the support arms and the vessel; therefore, it was believed that the flow path must somehow bend around the flange.

After the necessary codes had been developed, a channel theory calculation was performed on the RCS region to conclusively ascertain the mechanism for the head compartment dose. Fig. 7 shows a perspective plot of the resulting response-flux generated by FANG and Table 2 gives the integral of $\hat{D}(r, Z)$ over radius for each axial interval.

The perspective plots are very useful in interpreting $c(r, z)$. Fig. 7 clearly shows that two basic streaming paths exist in the lower RCS region and that these two components merge, forming a single component that flows between the reactor vessel and the vessel support arm. The peak that occurs between the $\mathrm{B}_{4} \mathrm{C}$ collar and the cavity shield is obviously a function of the gap-width between the support arms and the shield; however, a second, equally large peak lies between the vessel and steel 

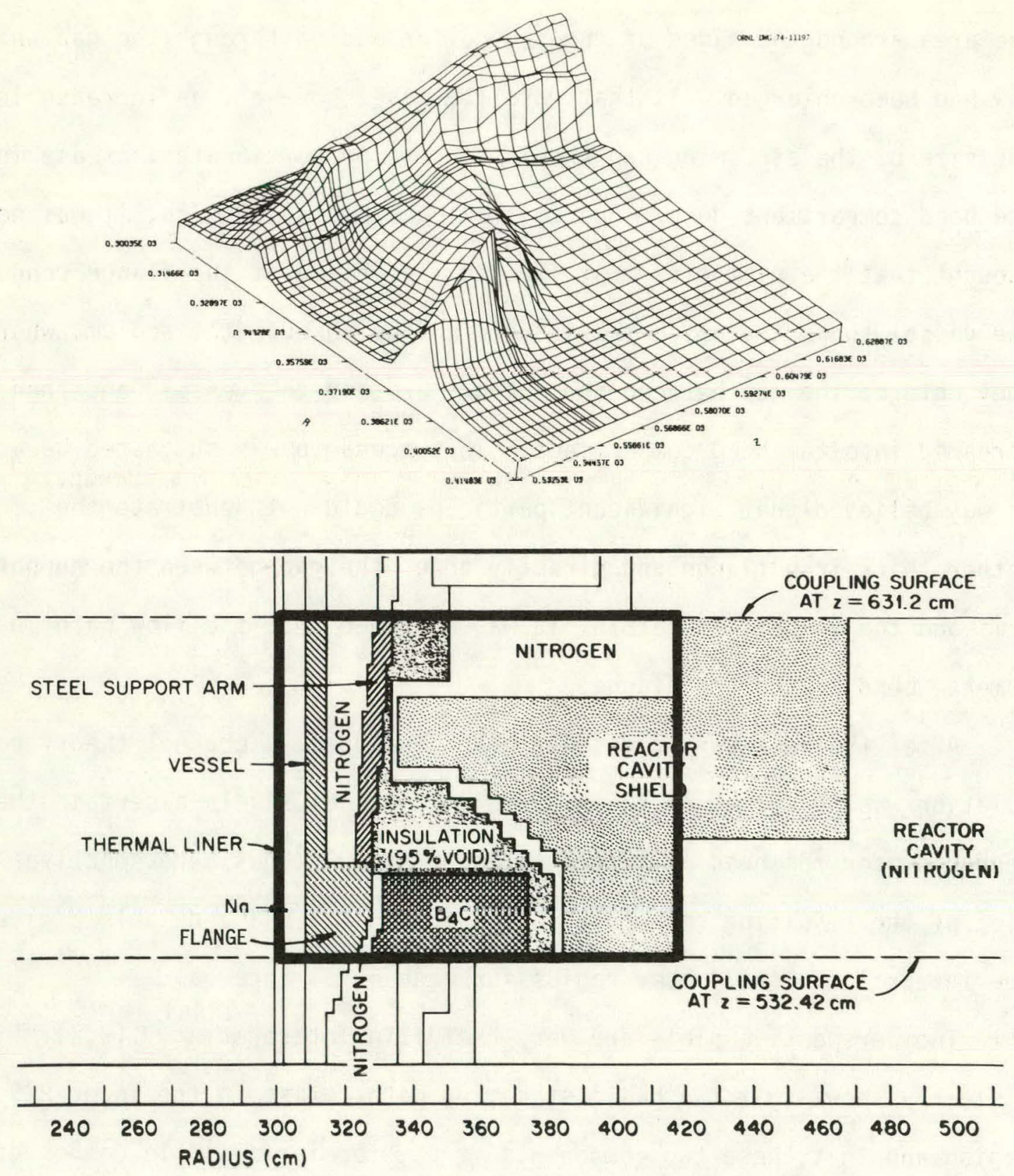

Fig. 7. Contributon Flux in RCS Region of FTR 
Table 2. Contributon Leakage Integrated over $r$ in FFTF RCS Geometry

\begin{tabular}{cc}
\hline Axial Coordinate & Response $\left(\mathrm{mr} / \mathrm{hr}-\mathrm{cm}^{3}\right)$ \\
\hline 26 & $8.96+03$ \\
25 & $8.79+03$ \\
24 & $8.59+03$ \\
23 & $8.58+03$ \\
22 & $8.55+03$ \\
21 & $8.46+03$ \\
20 & $8.26+03$ \\
19 & $8.05+03$ \\
18 & $7.85+03$ \\
17 & $7.90+03$ \\
16 & $7.99+03$ \\
15 & $8.19+03$ \\
14 & $8.52+03$ \\
13 & $8.63+03$ \\
12 & $9.11+03$ \\
11 & $9.28+03$ \\
10 & $9.54+03$ \\
9 & $9.53+03$ \\
8 & $9.45+03$ \\
7 & $9.43+03$ \\
6 & $9.13+03$ \\
5 & $8.61+03$ \\
4 & $1.30+04$ \\
3 & $1.23+04$ \\
2 & $1.17+04$ \\
1 & $1.13+04$ \\
& \\
\hline &
\end{tabular}

${ }^{*}$ Read as $8.96 \times 10^{3}$. 
support arm, and this streaming is unaffected by changes in the reactor cavity shield.

The figure also shows that the response-flux flows directly through the steel flange, not around it as was earlier suspected. Physically, this indicates that even though the neutron flux is well attentuated as it passes through the flange, particles that do survive contribute heavily to the head compartment dose. Mathematically, the peak is due to the high adjoint flux in this region, and folding the adjoint with even a Emall forward flux can pruduce d large contribution to the dose. On the other hand, the peak lying between the $\mathrm{B}_{4} \mathrm{C}$ and RCS is due to $\mathrm{a}$ large forward flux and only a moderately high adjoint, Channel thenry thus provides information which is not available from either the forward or adjoint calculation alone.

Since the particles comprising the two peaks can never be lost, they must necessarily flow together above the cavity shield, summing to create the single, large ridge in the upper left quadrant, which represents the channel of major dose contribution. The valley located between $r-$ $329 \mathrm{~cm}$ and $r-370 \mathrm{~cm}$ corresponds to the $\mathrm{B}_{4} \mathrm{C}$ collar, and indicates its effectiveness in lowering head compartment dose. Also, the flat area in the upper, right quadrant is a plain of low response-flux, since the reactor cavity shield is located at that position.

Table 2 again illustrates the integration of $\hat{D}(r, z)$ to obtain a response functional; in this case, it is the intcgrated neutron duse ill the head compartment. The values in this example are not as constant as in the first example due to the greater anisotropy in the neutron flux and the corresponding increase in difficulty which FANG encounters 
in reconstructing angular flux from only $P_{3}$ moments. The coarser mesh size also affects the accuracy. However, if a mean response value of 9.2 is chosen and divided by the adjoint source volume located in the head compartment, an average head compartment dose rate should be obtained. The resulting value is $.042 \mathrm{mr} / \mathrm{hr}$, which compares well with $.039 \mathrm{mr} / \mathrm{hr}$ found in a forward calculation. Thus, even for this rather difficult example, the $P_{3}$ moments appear to at least give good qualitative results for the observable.

\section{CONCLUSION}

The spatial channel theory technique appears to have great potential in two-dimensional analysis of complex systems. It can furnish additional insight into the radiation transport process which is not available from either a forward or adjoint calculation by itself. The increased understanding of the streaming paths in a reactor eliminates guess work in determining optimum placement of shielding, and also aids in the evaluation of shields already designed.

In most conventional shielding computation today, forward and adjoint fluxes are readily available, and once they are obtained, the channel theory calculation is inexpensive, and can actually save money by avoiding costly trial-and-error computer runs. As an example, at ORNL the standard shielding code is DOT, which required approximately 440 CPU minutes on an IBM $360 / 91$ computer to calculate forward and adjoint fluxes for the RCS region (Step 2) of the FFTF that was discussed 
in this article. In comparison, FANG took only 2 CPU minutes for a complete channel theory analysis of the same region. Obviously, channel theory is not restricted to DOT-FANG computation, and can be applied with equal success to any geometry encountered in shielding analysis for which forward and adjoint fluxes have been obtained.

Channel theory calculations are now a part of the standard procedure in the ORNL Shielding Analysis Program. The technique has already been applied sur.ressfully in FFTF studies and will be uscd in future shielding work on the $\mathrm{Cl}$ inch River Breeder Reactor Project, the Gas-Cooled Fast Reactor Program, and in analysis of shielding experiments. The area of thermal reactor shielding should also benefit from this type of analysis, and hopefully channel theory will be applied to the difficult streaming problems encounted in commercial design.

\section{ACKNOWLEDGMENTS}

The authors extend their appreciation to J. H. Marable for his discussion concerning the theoretical development of this work, and to Susan Y. Marler for her patience and effort in typing the manuscript. 


\section{REFERENCES}

1. Lorraine S. Abbott and F. R. Mynatt, "Review of ORNL Radiation Shielding Analyses of the Fast Flux Test Facility Reactor," ORNL5027, July, 1975.

2. H. Goldstein, et al, "Theoretical Studies of the Sensitivity of Neutron Transport in Iron to Cross-Section Properties, "Paper No. D2, Fourth International Conference on Reactor Shielding, Paris France, 1972.

3. W. W. Engle, Jr. and F. R. Mynatt, "A Technique to Determine the Shape of a Minimum Weight Shield," Transactions, 17:556, ANS San Francisco Conference, November 11-15, 1973.

4. M. L. Gritzner, W. W. Engle, Jr., and F. R. Mynatt, "FACT - A Computer Program for Spatial Coupling of Forward and Adjoint TwoDimensional Discrete Ordinates Calculations," ORNL-5167, To be Published.

5. J. Lewins, "Variational Representations in Reactor Physics Derived from a Physical Principle," Nuclear Science and Engineer-ing, 8:95 1960 .

6. G. I. Bell and S. Glasstone, Nuclear Reactor Theory, Van Nostrand Re inhold Company, 1970.

7. W. A. Rhoades and F. R. Mynatt, "The DOT III Two-Dimensional Discrete Ordinates Transport Code," ORNL-TM-4280, September, 1973.

8. M. L. Williams, "The FANG Angular Folding Code for Channel Theory Analysis," ORNL-TM-5228, To Be Published.

9. N. M. Greene. et al, "AMPX - A Modular Code System for Generating Coupled Multigroup Neutron Gamma Libraries from ENDF/13," ORNL-TM$3706,1974$.

10. W. W. Engle, Jr., M. B. Emmett, and M. L. Williams, "Analys is of the Complex Reactor Cavity Shield in the FTR," Transactions, 22:783, ANS San Francisco Conference, November 16-21, 1975. 